\title{
Adaptive Kinetic Architecture and Collective Behavior: A Dynamic Analysis for Emergency Evacuation
}

\author{
Angella Johnson ${ }^{1, *}$, Size Zheng ${ }^{2}$, Aiichiro Nakano ${ }^{3}{ }^{\mathbb{D}}$, Goetz Schierle ${ }^{1}$ and Joon-Ho Choi ${ }^{1}$ \\ 1 Building Science, School of Architecture, University of Southern California, Los Angeles, CA 90007, USA; \\ schierle@usc.edu (G.S.); joonhoch@usc.edu (J.-H.C.) \\ 2 Chemical Engineering, Viterbi School of Engineering, University of Southern California, \\ Los Angeles, CA 90007, USA; sizezhen@usc.edu \\ 3 Collaboratory For the Advanced Computing and Simulations, University of Southern California, \\ Los Angeles, CA 90007, USA; anakano@usc.edu \\ * Correspondence: angelajo@usc.edu
}

Received: 3 January 2019; Accepted: 7 February 2019; Published: 13 February 2019

\begin{abstract}
Adaptive kinetic architecture has emerged from a need for innovative designs that adapt to the environment and changing needs of the occupants. Architectural design and modes of egress are critical in an emergency. Flocking describes a certain collective behavior where agents are brought together in groups and move as a cohesive unit from place to place. Collective behavior may be observed in microscopic as well as macroscopic environments. Crowd modeling incorporates the study of human behavior, mathematical modeling, and molecular or fluid dynamics. The simulation of agents and their movement in the built environment is beneficial for design professionals, scientists, and engineers. Human behavior in panic situations is notably similar to fluids and molecules. The objective of this research was to evaluate the movement of agents in buildings using discrete dynamic simulation. We used a novel discrete molecular dynamics technique to simulate the evacuation of agents in panic situations. Various adaptive geometric configurations were analyzed for improved crowd flow. Kinetic walls were modeled in order to evaluate design optimization as it relates to rates of egression. This research proposes the use of kinetic walls to improve safety and efficiency during an emergency evacuation. Adaptive geometric configurations show improvements over the conventional design framework.
\end{abstract}

Keywords: adaptive design; kinetic architecture; collective behavior; energy efficiency; sustainability; egress; discrete molecular dynamics

\section{Introduction}

Kinetic architecture has emerged from a need for innovative designs that adapt to the environment and changing needs of the occupants. It is kinetic in the sense that it possess the ability to adjust to its environment which forms an ecological system [1-3]. It is ecological because it can be likened to a living organism and its physical surroundings. In addition to having improved aesthetics, kinetic structures play an important role in environmental sustainability. Kinetic architecture is symbiotic in nature [4]. The concept of responsive structures encompasses energy efficiency with a focus on heating, cooling, light, sound, and ventilation. Adaptive buildings do not require human assistance but respond naturally to the environment in impressive ways, therefore reducing energy consumption. Architectural features include, but are not limited to, movable fenestration and responsive facades. Currently, software tools and innovative designs are being utilized to reach sustainability goals of zero-energy buildings [5-8]. Zero-energy buildings are designed to consume only as much energy that is needed an that can be produced by onsite sources over a specified time. These sources include renewable energy geration like solar power. The Al Bahar Towers, located in the city of Abu Dhabi, is known for its innovative and 
sustainable design. Its shading device consists of a series of transparent components which open and close in response to the Sun's trajectory. The two towers have more than 1000 individual solar protectors, which are controlled by the building's management system. The Al Bahar Towers were designed by Aedas Arquitectos studio and meets the 2030 Development Plan for Abu Dhabi. The architectural design is suitable given the climate in Abu Dhabi, which has very little rainfall and almost year-round sunshine. The climate in this region is subtropical and arid. In collaboration with Arup Engineers, Aedas' architects created large geometric patterns that form a sustainable and engaging facade. The facade system provides an adaptive and dynamic solution to the climate in this region (see Figure 1).

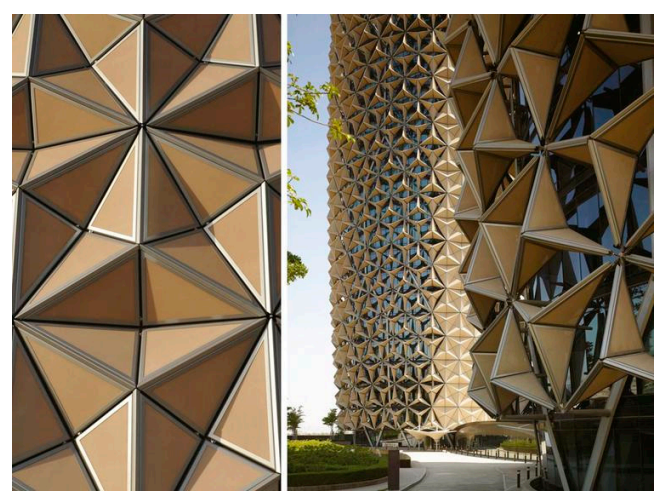

Figure 1. Al Bahar Towers.

Another example of this is the Milwaukee Art Museum designed by Santiago Calatrava, a Spanish architect, structural analyst engineer, and artist. The museum was designed with the comfort of the museum-goers in mind. It takes advantage of natural sunlight, ventilation, and radiant heating, which saves time and money (see Figure 2).

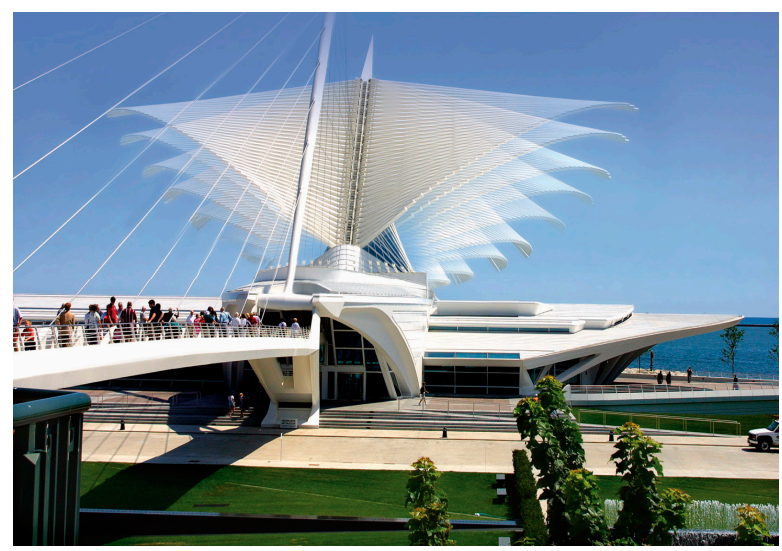

Figure 2. Milwaukee Art Museum.

Innovative designers, architects, and others in the construction community utilize multiple software tools to reach sustainability goals $[9,10]$. The concept of adaptive structures for safety has not been thoroughly explored. It is postulated in this research that kinetic architecture might be used for additional purposes other than energy efficiency. In particular, adaptive structures can incorporate the safe egress of large numbers of occupants. If implemented, adaptive kinetic architecture might be considered for areas with large gatherings, such as museums and concert halls. With this concept in mind, a building may be designed in such a way that parts of the structure move without affecting the structural integrity. In theory, adaptive kinetic structures are well suited for gathering places, such as museums and concert halls. With this concept in mind, buildings may be designed in such a way that parts of the structure move without affecting the structural integrity. This study investigated the collective behavior of crowds and methods of improving flow by utilizing discrete molecular dynamic simulation of adaptive walls. 


\section{Background}

Scientists Alder and Wainwright simulated elastic collision between hard spheres during the mid-1950s. Similarly, Rahman simulated liquid argon using the Lennard-Jones potential in the 1960s, which was a novel simulation at the time. Molecular dynamics gained popularity in material science and has since been utilized in biophysics, chemistry, and proteomics [11-13]. It is based on simulation techniques that involve interacting atoms, their equations of motion, and time evolution. Theoretically, it follows the laws of classical mechanics. Newton's Second Law of Motion:

$$
\mathrm{F}_{n}=\mathrm{m}_{n} \mathrm{a}_{n}
$$

where $\mathrm{F}_{n}$ represents the force due to interacting atoms, $\mathrm{m}_{n}$ is the mass of the atom, and $\mathrm{a}_{n}=\mathrm{d}^{2} \mathrm{r}_{n} / \mathrm{dt}^{2}$.

The N-body problem is insoluble for three or more bodies and originated in the study of the solar system. In the above Equation (1), each $n$ in the system constitutes $\mathrm{N}$ atoms. The nonquantum behavior of matter can be mostly understood by classical terms. At the microscopic level, classical N-body problems of matter can be understood. Various modeling techniques have been developed for molecular-level research, including molecular modeling, Monte Carlo methods, cellular automata, the lattice Boltzmann method, molecular dynamics with electron density function theory, and quantum-based techniques, to name a few [14-16]. Quantum mechanics is based on a measure of uncertainty-the uncertainty principle. On the other hand, the momentum and position can be determined at all times with molecular dynamics. Molecular dynamics is therefore deterministic.

A deterministic system is one in which there is no randomness. In principle, given a set of initial conditions (position and/or velocity), the time evolution can be determined. This model will produce the same output given the same initial state. Time evolution is the process by which time passes. There is an internal change of state. In a continuous model the variables change in a continuous way, but not in a predictable way. In this way, it is not possible to predict exactly what will happen.

Equations of motion are solved numerically. The Lennard-Jones potential was proposed by Sir John Edward Lennard-Jones. This potential describes the interaction between molecules and non-bonding atoms. This equation accounts for attractive and repulsive forces and takes into consideration the distance of separation. Attractive forces may be dipole-dipole, London interactions, or dipole-induced dipole interactions. The 12-6 Lennard-Jones potential model consists of two parts-a repulsive term $\left(\frac{\sigma}{r}\right)^{6}$ and an attractive term $\left(\frac{\sigma}{r}\right)^{12}$ :

$$
\mathrm{V}(r)=4 \varepsilon\left[\left(\frac{\sigma}{r}\right)^{12}-\left(\frac{\sigma}{r}\right)^{6}\right]
$$

where $\mathrm{V}$ is the intermolecular potential, $r$ is the distance of separation between particles, $\varepsilon$ is the well depth bonding energy, and $\sigma$ is the distance.

The simplified form is:

$$
\mathrm{V}(\mathrm{r})=\frac{A}{r^{12}}-\frac{B}{r^{6}}
$$

where $A=4 \varepsilon \sigma^{12}$ and $B=4 \varepsilon \sigma^{6}$.

The Lennard-Jones equation is a function of the distance between the centers of both particles. The distance between the centers of the particle is bound, so their centers are drawn together until the particles reach equilibrium. If the particles are pressed further together, repulsion occurs. In this case, the bound particles are so close that the electrons orbiting their shell are forced to occupy the other particle's shell. The potential energy becomes increasingly positive as the separation distance decreases below equilibrium. The opposite is true for negative potential energy due to the long separation distances. At these long distances, the pair of molecules experiences a stabilizing force. Molecular dynamics is a statistical mechanics method based on the partition function. The partition function is a function of temperature and other parameters, which describes the statistical properties in thermal equilibrium. Each type of partition function corresponds to different types of statistical 
ensembles. The grand canonical partition function applies to a canonical ensemble in which the system exchanges both particles and heat with the environment. This is accomplished with a fixed volume, temperature, and chemical potential (see Figure 3).

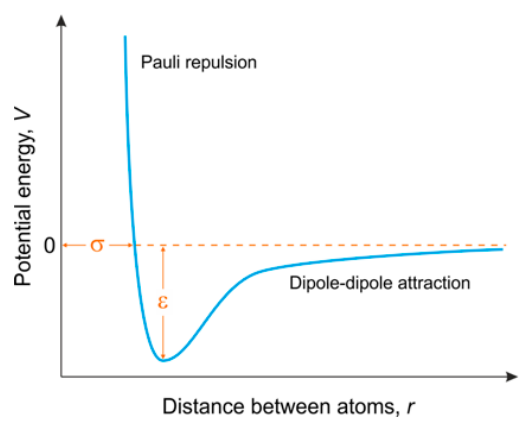

Figure 3. Lennard-Jones potential.

\section{Methodology}

The discrete molecular dynamics (DMD) simulation algorithm is an extremely fast alternative to traditional molecular dynamics. It was first introduced in 1959 by Alder and Wainwright for simulations of hard spheres. Later, it was used by Rapaport for the simulation of polymer chains. It has since been adopted for simulations of protein-like polymers. The benefits of using DMD are that it is extremely fast and suitable for the simulation of large systems on long timescales. During DMD simulation, potentials applied to particles are approximated by discontinuous step functions of the interparticle distance $r$ (Figure 4).
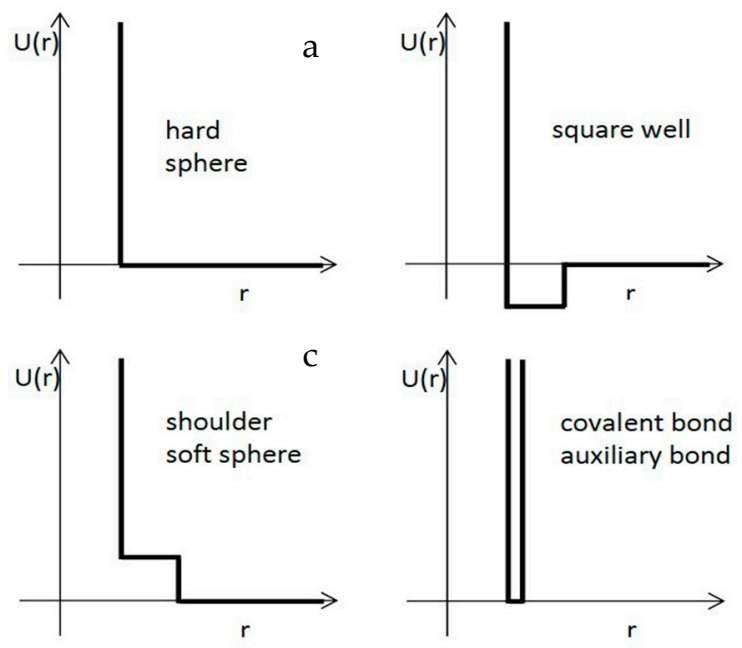

Figure 4. Discrete molecular dynamics (DMD) potential: (a) hard sphere collision, (b) attractive square well interaction, (c) repulsive soft interaction, and (d) covalent bond and auxiliary bond interaction.

No force is exerted on particles until their distance becomes equal to the point of discontinuity on the potential. When particles encounter a potential discontinuity, this is called an "event". Between events, particles move at constant velocities. Because the energy change during each event is known, the post-event velocities can be calculated by solving the conservation of momentum and the conservation of energy simultaneously. Thus, the trajectory of a particle can be simulated discontinuously between events.

The simulation code for DMD is significantly different from that for traditional molecular dynamics because the particles are moved discontinuously from one event to the next with known velocities. SDMD is a simulation package based on the DMD technique and a high-resolution all-atom molecular model. The package was written in the $\mathrm{C}$ language by Size Zheng and has since been optimized [17]. Its modularized structure is highly expandable: New molecular 
groups can be introduced by simply adding a new formatted connection and potential data files. Pedestrian simulation is important in predicting the escape time for emergency planning, which leads to safer buildings. Advances in traffic science have encouraged physicists and other scientists to investigate the evacuation process and pedestrian flow [18]. Evacuation dynamics and pedestrian flow are closely connected with the many-body particle system, which is a system of strongly interacting agents. Individual pedestrians can be mimicked by molecular dynamic theory.

Therefore, this research adopted a computational tool based on discrete molecular dynamics that has also been applied to chemical and biological systems. Even though it is commonly used for rigid-body mechanics, protein folding, and genome analysis, its technical features are applicable to the complexity of pedestrian interaction [19-21]. The simulation code for discrete molecular dynamics (DMD) is different from that for traditional molecular dynamics. Its modularized structure is highly expandable to include various molecular groups. Currently, the package is able to run simulations under various situations as follows:

- NVT and NVE ensembles (constant number, volume, temperature) and (constant number, volume, energy), respectively;

- bulk conditions as well as parallel pores, cylindrical tubes, and spherical cavities;

- replica exchange molecular dynamics (REMD) simulation;

- dynamic confined systems, as the confined walls expand, compress, and pulse; and

- molecule flow through a tube between two reservoirs.

Methods of analysis are as follows:

- Removal of periodic boundary conditions of trajectory;

- computation of the number of different types of hydrogen bonds;

- computation of the Ramachandran plot;

- computation of system temperature, kinetic energy, and potential energy;

- computation of the number of clusters during aggregation; and

- analysis of data from T-REMD by the weighted histogram analysis method (WHAM).

In order to apply sDMD to more complex systems, we upgraded the molecular and potential models from coarse-grained to all-atom models. Large amounts of code were rewritten and optimized.

\section{Results}

The main goal of this research was to evaluate the simulation of agents in buildings using discontinuous molecular dynamic simulation. We analyzed exit corridors, particulary areas where bottleneck occurs [22-24]. Responsive geometrical walls were analyzed to resemble wall units commonly found at points of egression. Discontinuous or discrete molecular dynamic simulation was utilized for optimal evacuation methodology. Four configurations were simulated as shown in Figure 5. The configurations were as follows: (a) Reference configuration, nothing added in the room; (b) kinetic contoured wall-a movable wall strategically placed in the middle of the room; (c) linear compression wall-the wall compresses toward the center; and (d) curved egression wall-a kinetic wall located at an entrance/exit to aid in egressing occupants. 

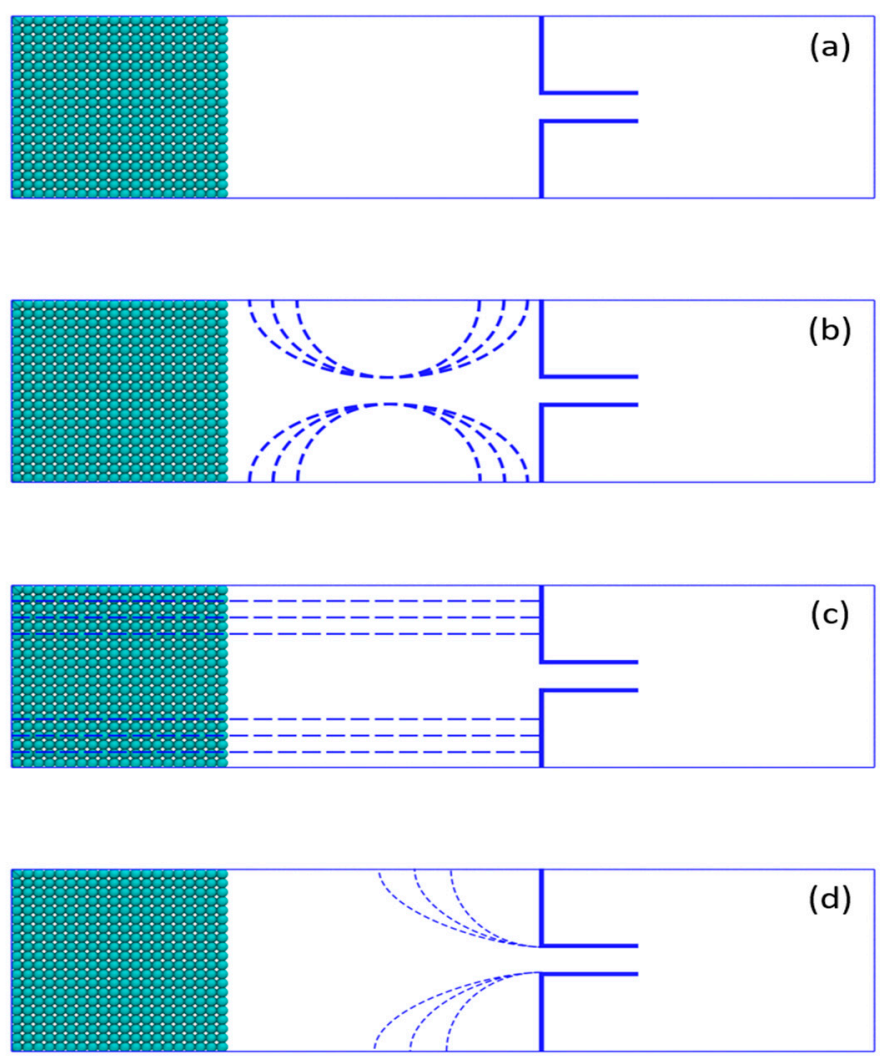

Figure 5. Simulation configurations.

Table 1 shows the varying wall types with their respective distances or radii.

Table 1. Configuration parameters.

\begin{tabular}{cccccccc}
\hline Wall Types & \multicolumn{7}{c}{ Distance/Radius (m) } \\
\hline Compression Wall & $\mathbf{4 . 0 5 0}$ & $\mathbf{4 . 7 2 5}$ & $\mathbf{5 . 4 0 0}$ & $\mathbf{6 . 0 7 5}$ & $\mathbf{6 . 7 5 0}$ & $\mathbf{7 . 4 2 5}$ & $\mathbf{8 . 1 0 0}$ \\
\hline Contour wall & 8.775 & 9.450 & 10.125 & 10.800 & 11.475 & 12.150 & 12.825 \\
Curved wall & 8.775 & 9.450 & 10.125 & 10.800 & 11.475 & 12.150 & 12.825 \\
\hline
\end{tabular}

Different configurations have different effects on escaping agents-where some cases may help, the others may not. The total evacuation time was recorded. Results are the averages of the 10 independent simulations. This is shown in Figure 6.

In Figure 6, the red line represents the reference configuration, pop represents the kinetic contour wall, cpr represents the linear compression wall, and cur represents the curvature wall. Analytical results show that compared with the reference configuration, the curved egression wall significantly reduced the evacuation time. The reduction in evacuation time was up to $14 \mathrm{~s}$. The linear compression wall showed some improvement, but in some cases would even increase the time cost. The kinetic contour wall, however, performed disappointingly in every case. It would, in a real situation, prevent a crowd from escaping and in many cases increase the escape time.

Figures 6 and 7 show that if we did nothing (represented by the reference situation (red line)), there would be approximately seven people left in the room after $240 \mathrm{~s}$. In the case of the compression wall, it may save one or two more people. The curved egression wall construction showed a reduction in the number of casualties (only one or two casualties). This would save as many as six people compared to the other cases. However, if we use the kinetic contour wall configuration, more people would remain trapped in the room, increasing the total number of casualties to 12 . This is almost double the number in the reference case. 


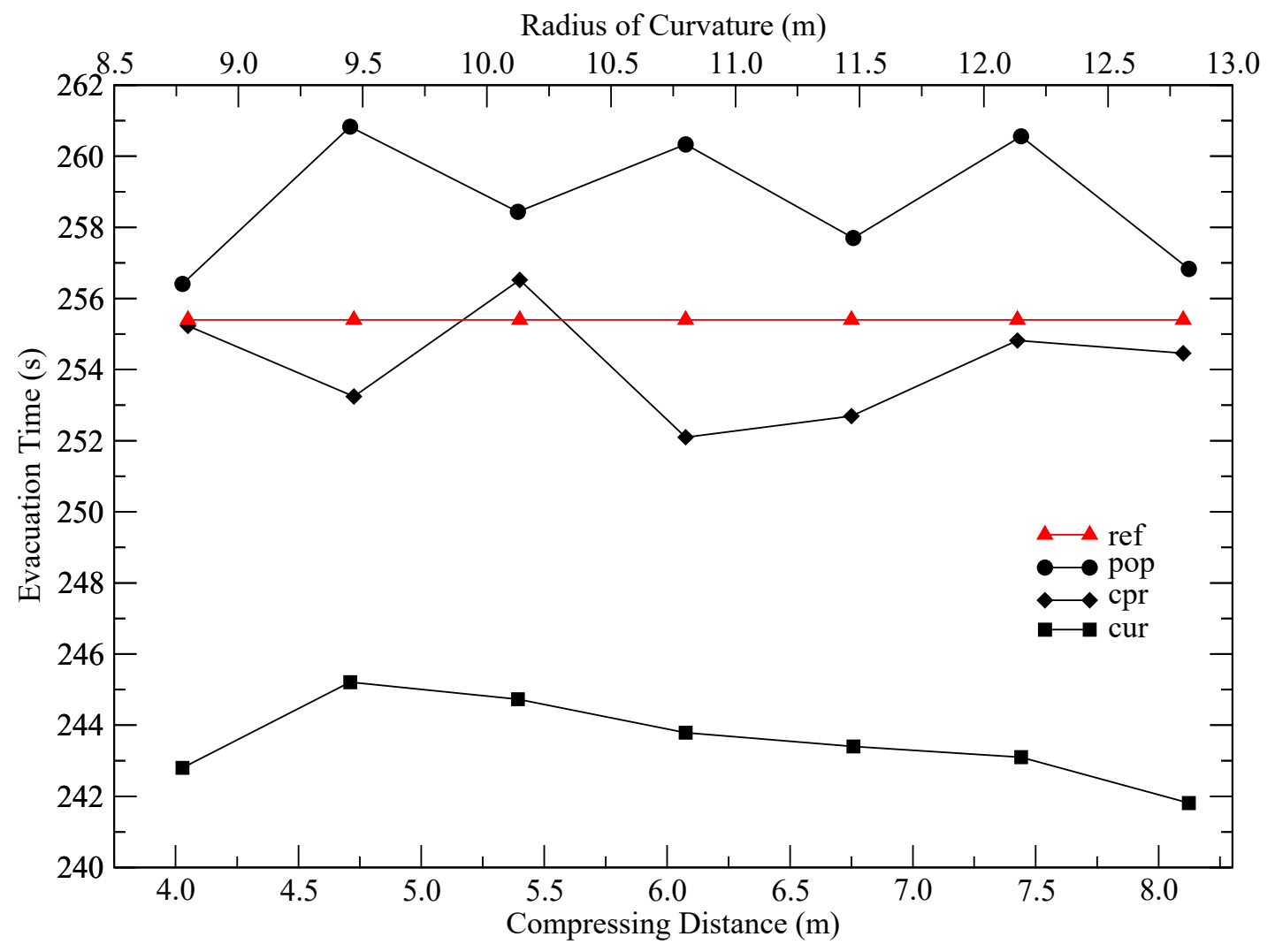

Figure 6. Escape time for different configurations. Abbreviations: ref-reference configuration, pop-kinetic contour wall, cpr-linear compression wall, and cur-curvature wall.

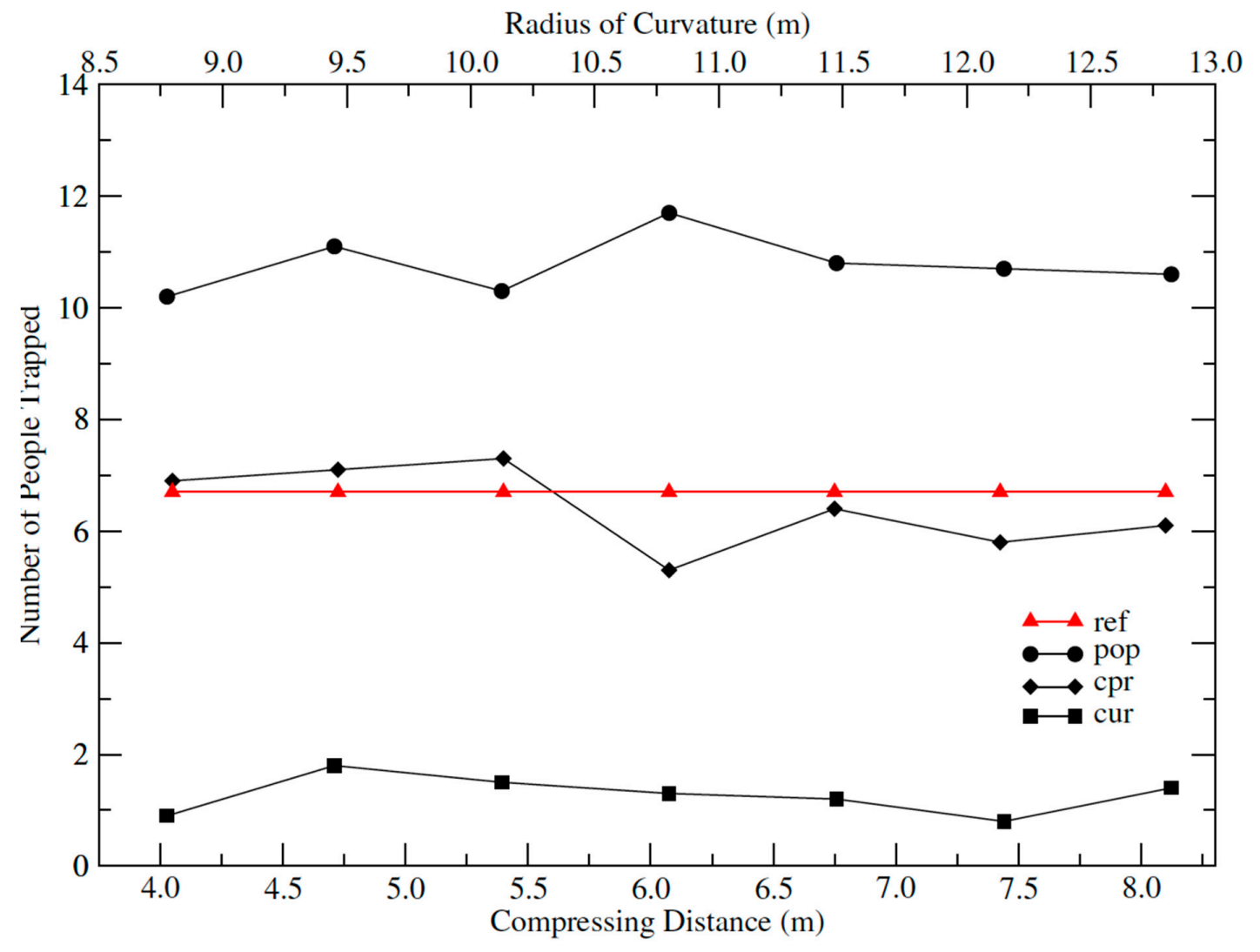

Figure 7. The number of people trapped in the room after $240 \mathrm{~s}$ of evacuation time. 


\section{Discussion}

Throughout the history of architecture there has been a wide range of design principles. Designs of culturally specific, fixed, or immobile structures can be found in much of the built environment. Successfully navigating exits or entrance ways is crucial in emergency evacuation. This is evident in emergency situations where individuals lose the ability to orient to their surroundings, panic, and fail to act logically. Instead of responding independently, individuals rely on group action. In this situation, the crowd or collective whole possesses knowledge of avoiding dangerous situations. Individual cognitive abilities are lost. The result is a groupthink mentality which may not be optimal for safety. If the danger is heightened, assertive behaviors, such as pushing or shoving, are exhibited. This behavior is termed as non-adaptive crowd behavior. In a familiar environment, pedestrians show some basic attributes. The most basic principle is the principle of least effort. This principle postulates that people or animals will naturally choose the path of least effort or resistance $[25,26]$. The action of a pedestrian in a normal situation suddenly changes in an emergence situation. For example, the desired velocity increases as occupants attempt to leave a building as quickly as possible and this leads to a typical formation. Occupants who are not well acquainted with an area will tend to exit where they entered. This is the case even though another exit might exist or be easier to reach. They might therefore lose the ability to orient themselves to the environment and exhibit flocking or herding behavior [27]. This behavior is responsible for injury and death in herding disasters [28-31]. The behavior of crowds in emergency situations can be challenging to analyze since an actual experiment involves exposing participants to a possibly hazardous environment. Drills, such as fire or earthquake drills, are effective but can hardly recreate panic situations. Crowd behavior has been studied for decades and evacuation methods have been developed with the aid of time-lapse films, observations, and simulations [32,33]. Inspired by the computer gaming, entertainment, and movie industries, researchers use familiar techniques to model agents in architectural buildings [34].

Biomimicry, or the patterning of nature, utilizes sustainable solutions for challenges faced by humanity. Flocking or herding describes certain collective behavior where species are brought together and move as a cohesive group from place to place [35,36]. Human behavior in panic situations is notably similar to herding and flocking. Interestingly enough, these movements are also similar to those of fluids and certain molecules [37-39]. What is observed at the macroscopic scale is also observed at the microscopic scale. Collective behavior may be observed in the microscopic as well as the macroscopic environment [40-43].

The discontinuous molecular dynamics method was used to simulate an evacuation process. During the simulations, 400 people (modeled as spheres) moved toward the exit door. The driving force was simulated as a potential well, where the minimal was located near the exit. The random movements of the spheres followed Brownian motions. The room had dimensions of $40 \times 16(\mathrm{~L} \times \mathrm{W}) \mathrm{m}^{2}$. The exit door was $3 \mathrm{~m}$ wide. Four configurations were simulated as shown in Figure 8. The configurations are as follows: (a) Reference configuration, (b) kinetic contoured wall, (c) linear compression wall, and (d) curved egression wall-kinetic wall located at an the entrance/exit. For configurations (b), (c), and (d), seven different parameters were used. Configurations (b) and (d) had different radii of curvature. In the case of (c), different compressing distances were used. 

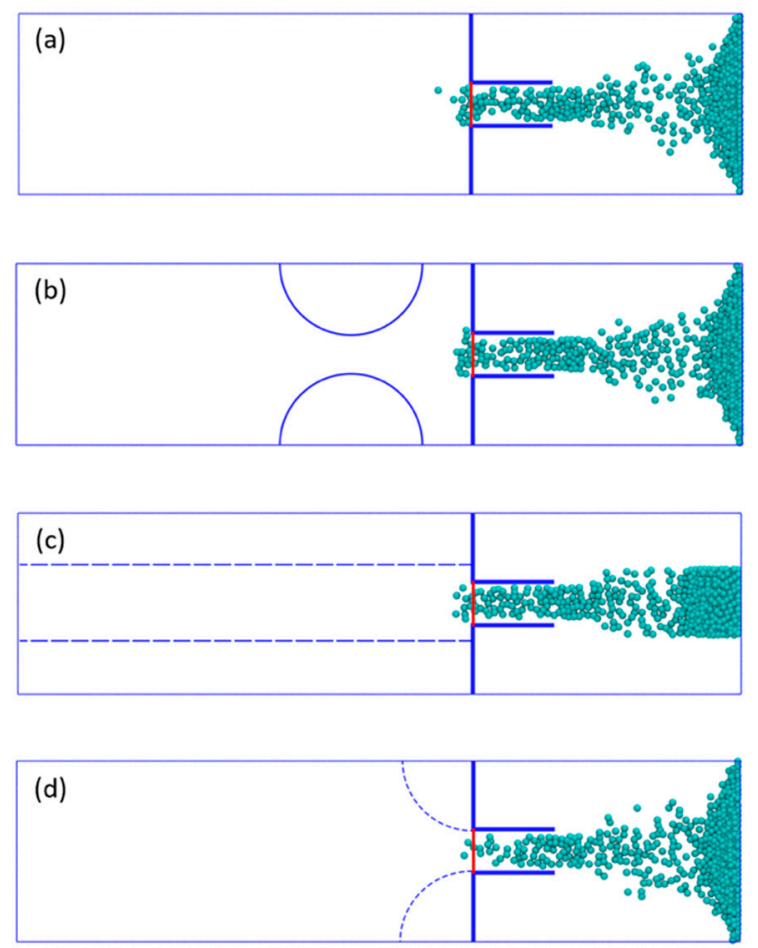

Figure 8. Snapshots of the evacuation process at $240 \mathrm{~s}$ for different configurations: (a) Reference, (b) kinetic contour wall, (c) linear compression wall, and (d) curved egression wall.

We found that different configurations resulted in different times for agents escaping. In some cases this was shown to help, where as in others it did not. In each configuration, we performed 10 independent simulations in order to model the evacuation process for up to $420 \mathrm{~s}$. The total evacuation time was recorded. Results are the averages of the 10 independent simulations. Analytical results, when compared with the reference configuration, show that the curved egression wall significantly reduced the evacuation time. The reduction in evacuation time was up to $14 \mathrm{~s}$. The linear compression wall showed some improvement although it increased the time cost. The kinetic contour wall, however, performed disappointingly in every case. It would, in a real situation, prevent a crowd from escaping and in many cases increase the escape time. On the basis of the existing test case, if we set a fixed evacuation time at $240 \mathrm{~s}$, we can also obtain the number of agents that would remain trapped in the respective rooms. The simulation snapshots are shown in Figure 8.

Preliminary results show that evacuation time is improved with a combination of changes in the geometric space at egression. For this research we outlined and quantitatively compared various architectural layouts. We described the strong correlation between event-driven molecular dynamics and the occupants' movement. From the microscopic view of molecular dynamics, we found applications to crowd simulation where agents are supported by observations and refined in computer simulation [44,45]. Discontinuous dynamic molecular modeling was utilized to optimize kinetic walls and occupants' egression. Kinetic structures have been shown to improve modes of egression of building occupants. The evacuation time, distribution of total distance, and kinetic wall configurations were compared. It is postulated that an effective crowd simulation could converge to form a simulation metric that depicts observable real-world data. As knowledge expands and modeling techniques improve, we will be able to learn more about social forces and the psychological and behavioral science of large crowds [46-48]. The proposed kinetic contour wall may show an improvement over the other architectural configurations because curved geometrical spaces reduce the shear-stress vector. Additionally, the results depict a streamlined pattern that is consistent with fluid flow through curved spaces. Fluid flow around curved geometries is encountered in much of nature from microscopic organisms to the cosmological scale. Researchers Debus, Mendoza, Succi, and Herrmann detailed in 
their journal article, "Energy Dissipation in Flows through Curved Spaces", that energy dissipation plays a significant role in fluid dynamics in curved spaces [49]. Curvature-induced viscous forces are shown to cause appreciable energy dissipation.

As a result of the research, we propose the use of curved kinetic obstacles at the point of egress. In comparing and analyzing the various configurations, the proposed kinetic wall (curved egression wall) performed well in all cases. An example of the proposed kinetic wall is shown in Figure 9.

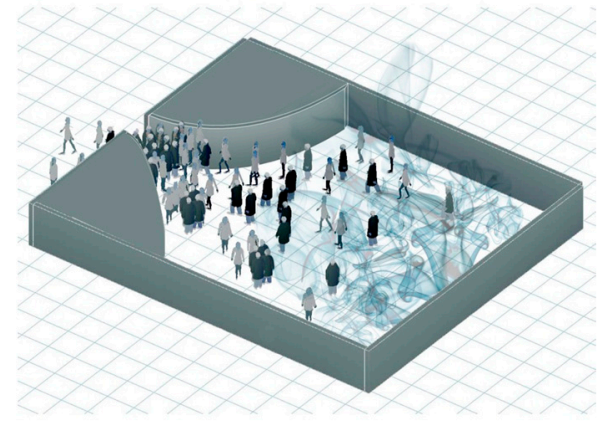

Figure 9. Proposed curved kinetic egression wall.

We compared the evacuation time, distribution of total distance, and evacuation rate. It is postulated that an effective crowd simulation could converge to form a simulation metric that depicts observable real-world data [50].

\section{Conclusions}

Understanding the interface between architecture, engineering, and behavioral science is important. This area of research spans many relevant topics and working together may better equip key individuals to address issues of safety and sustainability. For this research we outlined and quantitatively compared various architectural layouts. We analyzed the strong correlation between molecular dynamics and occupants' movement. From the microscopic view of molecular dynamics, we found applications to crowd simulation where agents are supported by observations and refined in computer simulation. Discontinuous dynamic molecular modeling was utilized in order to optimize kinetic walls and occupant's egression. Various architectural layouts have been shown to improve the modes of egression of building occupants. We compared the evacuation time, distribution of total distance, and kinetic wall configurations. Public structures, where large numbers of agents gather, may benefit from safety design features. Sustainable adaptive architecture, especially in corridors, hallways, and doors, can play a major role in assisting occupants to safely egress in emergency situations. In the near future, design professionals and engineers can design with crowd mobility and evacuation in mind. In the future, structures should be not only energy efficient but also safe and movable. There are a growing number of smart materials available for implementation. We propose a fire-retardant, kinetic egression wall that will aid occupants when evacuating in an emergency. As knowledge expands and modeling techniques improve, we will be better equipped to utilize our collective intelligence for smarter buildings and structures.

Author Contributions: The individual contribution of the authors was as follows: Conceptualization: A.J.; Data Curation: S.Z., Formal Analysis: A.J., S.Z.; Investigation: A.J.; Writing original draft: A.J.; Software: S.Z.; Review and editing of manuscript, G.S., J.C., A.N.; Visualization: A.J., S.Z.; Supervision: A.N., J.C., G.S.; Project administration: A.N.

Funding: This research received no external funding.

Acknowledgments: The research for this paper was made possible by a partial support from the Gesundheit Family and Architectural Guild Graduate Traveling Fellowship and the University of Southern California.

Conflicts of Interest: The authors declare no conflict of interest. 


\section{References}

1. Megahed, N.A. Understanding Kinetic Architecture: Typology, Classification, and Design Strategy. Archit. Eng. Des. Manag. 2017, 13, 130-146. [CrossRef]

2. Sanchez-del-Valle, C. Adaptive Kinetic Architecture: A Portal to Digital Prototyping. In Proceedings of the 2005 Annual Conference of the Association for Computer Aided Design in Architecture, Savannah, GA, USA, 13-16 October 2005; pp. 128-140.

3. Elkhayat, Y.O. Interactive Movement in Kinetic Architecture. J. Eng. Sci. 2014, 42, 816-845.

4. Sijakovic, M.; Peric, A. Symbiotic Architecture: Redefinition of Recycling Design Principles. Front. Archit. Res. 2018, 7, 67-79. [CrossRef]

5. Razaz, Z.E. Sustainable Vision of Kinetic Architecture. J. Build. Appraisal 2010, 5, 341-356. [CrossRef]

6. Wang, J.; Beltran, L.O.; Kim, J.H. From Static to Kinetic: A Review of Acclimated Kinetic Building Envelopes. World Renew. Energy Forum Proc. Sol. Conf. 2012, 5, 4022-4029.

7. Nagy, Z.; Svetozarevic, B.; Jayathissa, P.; Begle, M.; Hofer, J.; Lydon, G.; Wilmann, A.; Schlueter, A. The Adaptive Solar Façade: From concept to prototypes. Front. Archit. Res. 2016, 5, 143-156. [CrossRef]

8. Cudzik, J.; Nyka, L. Reasons for Implementing Movement in Kinetic Architecture. IOP Conf. Ser. Mater. Sci. Eng. 2017, 245, 042073. [CrossRef]

9. Heinze, M.; Voss, K. Goal: Zero Energy Building Exemplary Experience Based on the Solar Estate Solarsiedlung Freiburg am Schlierberg, Germany. J. Green Build. 2009, 4, 93-100. [CrossRef]

10. Aelenei, L.; Gonçalves, H. From Solar Building Design to Net Zero Energy Buildings: Preformance Insights of an Office Building. Energy Procedia 2014, 48, 1236-1243. [CrossRef]

11. Rapaport, D.C. The Art of Molecular Dynamics Simulation, 2nd ed.; Cambridge University Press: New York, NY, USA, 2004.

12. Berendsen, H.J.C. Biophysical Applications of Molecular Dynamics. Comput. Phys. Commun. 1987, 44, 233-242. [CrossRef]

13. Perricone, U.; Guiotta, M.R.; Lombino, J.; Parrino, B.; Cascioferro, S.; Diana, P.; Cirrincione, G.; Padova, A. An Overview of Recent Molecular Dynamics Applications as Medicinal Chemistry Tools for the Undruggable Site Challenge. MedChem Comm 2018, 9, 920-936. [CrossRef] [PubMed]

14. Chopard, B.; Dupuis, A.; Masselot, A.; Luthi, P. Cellular Automata and Lattice Boltzmann Techniques: An Approach to Model and Simulate Complex Systems. Adv. Complex Syst. 2002, 5, 103-246. [CrossRef]

15. Pang, T. An Introduction to Computational Physics, 2nd ed.; Cambridge University Press: New York, NY, USA, 2006.

16. Press, W.; Teukolsky, S.A.; Vetterling, W.T.; Flannery, B.P. Numerical Recipes, 3rd ed.; The Art of Scientific Computing; Cambridge University Press: New York, NY, USA, 2007.

17. Donev, A.; Torquato, S.; Stillinger, F. Neighbor List Collision-driven Molecular Dynamics Simulation for Nonspherical Hard Particles. I. Algorithmic Details. J. Comput. Phys. 2005, 202, 737-764. [CrossRef]

18. Lin, M.C.; Manocha, D. Virtual Cityscapes: Recent Advances in Crowd Modeling and Traffic Simulation. Front. Comput. Sci. China 2010, 4, 405-416. [CrossRef]

19. Proctor, E.A.; Ding, F.; Dokholyan, N. Discrete Molecular Dynamics. Comput. Mol. Sci. 2011, 1, 80-92. [CrossRef]

20. Emperador, A.; Solemou, A.; Sfriso, P.; Pons, C. Efficient Relaxation od Protein-Protein Interfaces by Discrete Molecular Dynamics Simulations. J. Chem. Theory Comput. 2012, 9, 1222-1229. [CrossRef] [PubMed]

21. Zheng, S. Discontinuous Molecular Dynamics (DMD)Simulation Package- sDMD User Manual, Version 0.95. Available online: https:/ / github.com/alexzheng42/sDMD (accessed on 8 February 2019).

22. Bagloee, S.A.; Sarvi, M.; Heshmati, M.; De Gruyter, C. Crowd Safety Through Architectural Design of Exit Corridors, Australasian Transport Research Forum 2015 Proceedings, ATRF, Australasian Transport Research Forum, Canberra, Australia. In Proceedings of the 37th Australasian Transport Research Forum, Sydney, Australia, 30 September-2 October 2015.

23. Hoogendoorn, S.; Daamen, W. Pedestrian Behavior at Bottlenecks. Transp. Sci. 2005, 39, 147-159. [CrossRef]

24. Kretz, T.; Grunebohm, A.; Schreckenberg, M. Experimental study of pedestrian flow through a bottleneck. J. Stat. Mech. Theory Exp. 2006, P10014. [CrossRef]

25. Moussaid, M.; Perozo, N.; Garnier, S.; Helbing, D.; Theraulaz, G. The Walking Behaviour of Pedestrian Social Groups and Its Impact on Crowd Dynamics. PLoS ONE 2010, 5, e10047. [CrossRef] 
26. Nagatani, T.; Nagai, R. Statistical Characteristic of Evacuation Without Visibility in Random Walk Model. Phys. A: Stat. Mech. Its Appl. 2004, 341, 638-648. [CrossRef]

27. Xiao, Z.; Ting, Z.; Meng, L. Modeling Crowd Evacuation of a Building Based on Seven Methodological Approaches. Build. Environ. 2009, 44, 437-445.

28. Helbing, D.; Farkas, I.; Vicsek, T. Simulating Dynamical Features of Escape Panic. Nature 2000, 407, 487-490. [CrossRef] [PubMed]

29. Helbing, D.; Johansson, A. Crowd and Evacuation Dynamics. In Encyclopedia of Complexity and Systems Science; Springer: New York, NY, USA, 2009. [CrossRef]

30. Lujak, M.; Giordani, S.; Ossowski, S. An Architecture for Safe Evacuation Route Recommendation in Smart Spaces. In Proceedings of the Ninth International Workshop on Agents in Traffic and Transportation (ATT 2016) Co-Located with the 25th International Joint Conference on Artificial Intelligence (IJCAI 2016), New York, NY, USA, 10 July 2016; Volume 1678.

31. Helbing, D.; Molnar, P.; Farkas, I.; Vicsek, T. Simulation of Pedestrian Crowds in Normal and Evacuation Situations. In Pedestrian and Evacuation Dynamics; Springer: Berlin, Germany, 2002; pp. 21-58.

32. Johansson, A.; Helbing, D.; Al-Abideen, H.Z.; Al-Bosta, S. From Crowd Dynamics to Crowd Safety: A Video-Based Analysis. Adv. Complex Syst. 2008, 11, 497-527. [CrossRef]

33. Kachroo, P.; Al-Nasur, S.J.; Wadoo, S.A.; Shende, A. Pedestrian Dynamics: Feedback Control of Crowd Evacuation; Springer: Berlin/Heidelberg, Germany, 2008. [CrossRef]

34. Silveira Jacques, J.C.; Musse, S.R.; Jung, C.R. Crowd Analysis Using Computer Vision Techniques. IEEE Signal Process Mag. 2010, 27, 66-77. [CrossRef]

35. Lissaman, P.B.S.; Shollenberger, C.A. Formation Flight of Birds. Science 1970, 168, 1003-1005. [CrossRef]

36. Gordon, D. What Do Ants Know That We Don't? Wired. July 2013. Available online: http: / / www.wired. com/2013/07/what-ants-yes-know-that-we-dont-the-future-of-networking/ (accessed on 6 July 2013).

37. Henderson, L.F. On the Fluid Mechanics of Human Crowd Motion. Transp. Res. 1974, 8, 509-515. [CrossRef]

38. Hughes, R.L. The Flow of Human Crowds. Annu. Rev. Fluid Mech. 2003, 35, 169-182. [CrossRef]

39. Guo, R.Y.; Huang, H.J. A Mobile Lattice Gas Model for Simulating Pedestrian Evacuation. Physica A 2008, 387, 580-586. [CrossRef]

40. Parisi, D.R.; Dorso, C.O. Microscopic Dynamics of Pedestrian Evacuation. Physica A 2006, 354, 606-618. [CrossRef]

41. Aveni, A. The Not-So-Lonely Crowd: Friendship Groups in Collective Behavior. Sociometry 1977, 40, 96-99. [CrossRef]

42. Vermuyten, H.; Beliën, J.; De Boeck, L.; Reniers, G.; Wauters, T. A Review of Optimisation Models for Pedestrian Evacuation and Design Problems. Saf. Sci. 2016, 87, 167-178. [CrossRef]

43. Helbing, D. A Fluid-Dynamic Model for the Movement of Pedestrians. Complex Syst. 1992, 6, $391-415$.

44. Liu, Z.; Liu, T.; Ma, M.; Hsu, H.; Ni, Z.; Chai, Y. A Perception-Based Emotion Contagion Model in Crowd Emergent Evacuation Simulation. Comput. Anim. Virtual Worlds 2018, 29, e1817. [CrossRef]

45. Helbing, D.; Molnar, P. Social Force Model for Pedestrian Dynamics. Phys. Rev. E 1995, 51, 5. [CrossRef]

46. Latane, B. The Psychology of Social Impact. Am. Psychol. 1981, 36, 343-356. [CrossRef]

47. Liu, Q. A Social Force Model for the Crowd Evacuation in a Terriost Attack. Phys. A 2018, 502, 315-330. [CrossRef]

48. Kang, J.; Jeong, I.; Kwun, J. Optimal Facility-final Exit Assignment Algorithm for Building Complex Evacuation. Comput. Ind. Eng. 2015, 85, 169-176. [CrossRef]

49. Debus, J.D.; Mendoza, M.; Hermann, S.H.J. Energy Dissipation in Flows Through Curved Spaces. Sci. Rep. 2017, 7, 42350. [CrossRef]

50. Haghani, M.; Sarvi, M. Crowd Behaviour and Motion: Empirical Methods. Transp. Res. Part B: Methodol. 2018, 107, 253-294. [CrossRef]

(C) 2019 by the authors. Licensee MDPI, Basel, Switzerland. This article is an open access article distributed under the terms and conditions of the Creative Commons Attribution (CC BY) license (http:/ / creativecommons.org/licenses/by/4.0/). 